\title{
Performance Improvement of DWDM System by incorporating the Concept of Polarization
}

\author{
Sruthi Subash $\mathrm{J}^{1}$, Jaini Sara Babu ${ }^{2}$ \\ ${ }^{l}$ (Optoelectronics and Communication Systems, TKM Institute of Technology, India) \\ ${ }^{2}$ (Electronics and Communication Engineering, TKM Institute of Technology, India)
}

\begin{abstract}
This paper demonstrate the design and analysis of $8 x 10 \mathrm{~Gb} / \mathrm{s}$ Dense Wavelength Division Multiplexing (DWDM) optical network by incorporating the concept of polarization. The performance of fiber optic network degrades due to nonlinear effects within the channel. Therefore in order the enhance the system capacity the crosstalk due to polarization have to be minimized. The two simple approaches being polarization interleaving and Polarization Multiplexing (PM). The results confirmed using eye diagrams that the system power signal loss reduces through adjacent channel polarization and also that the nonlinearities are polarization state dependent and best performance results are achieved when adjacent channels are launched with orthogonal polarization relative to each other.
\end{abstract}

Keywords: Dense wavelength Division Multiplexing, Polarization Multiplexing, Single mode Fiber, Wavelength Division Multiplexing.

\section{Introduction}

Optical communication has greatly influenced the communications environment towards the advanced information society. Innovations in optical fiber technology are enabling transmission of high-speed signals over transcontinental distances without the need for electronic regeneration. It has thus given thrust to networks with higher capacities and at lower costs. Wavelength Division Multiplexing (WDM) is one of the solutions to increase the capacity of the optical fiber using a different method from traditional Time Division Multiplexing (TDM) .In order to utilize the maximum bandwidth, trend is towards DWDM systems with the more number of channels with less wavelength spacing and large transmission distances [1].

In DWDM system, non-linear effects degrade the performance of the system. As the optical signal propagates through the fiber they undergo power fluctuations and crosstalk between the channels in DWDM system [2]. The dominant degradation effect of Cross Phase Modulation causes a nonlinear polarization dependent phase shift between the signals propagating through the same optical fiber [3]. This leads to polarization rotations even in the absence of polarization mode dispersion. Due constant phase shift and polarization rotations produced by XPM additional spectral components are generated in the optical spectra leading to polarization scattering which depolarizes the two polarization components and causes crosstalk between the $\mathrm{X}$ and $\mathrm{Y}$ polarization components.

The goal of this paper is to reduce the nonlinear impairments due to cross polarization by introducing the concepts of polarization interleaving and polarization multiplexing into the DWDM system. Sending signals in disjoint frequency bins on different optical carrier frequencies is called wavelength-division multiplexing. Such signals are orthogonal and individual bit streams can be recovered using optical band pass filters or electronic filters following a coherent receiver front-end. If signals leak energy into neighboring frequency bins, orthogonality is degraded and perfect reconstruction is no longer possible ('WDM crosstalk'). A possible counter-measure, which has been used, is alternating the polarization of adjacent channels to re-establish orthogonality in the polarization dimension (polarization interleaving). The second approach being polarizationdivision multiplexing in which one sends two independent signals on both orthogonal polarizations supported by a single-mode optical fiber. In order to recover these polarization-multiplexed bit streams, either a polarization beam splitter whose axes are constantly kept aligned with the signal polarizations (polarization control) can be used, or the detection of two arbitrary orthogonal polarizations (polarization diversity) using coherent detection can be employed [4],[5].

\section{System Architecture}

The block diagram for a polarization based DWDM system is shown in the Fig 1.In this concept the polarization state of adjacent channels have been varied where the transmitters with odd channel numbers are transmitted without changing their polarization, whereas the polarization is rotated by an angle of 90 degree for even channels. The system shown has $\mathrm{N}$ channels corresponding to 8 wavelengths. The power meters placed along the link indicates the reduction in optical power along the link which is an estimation of nonlinear impairment compensation. 


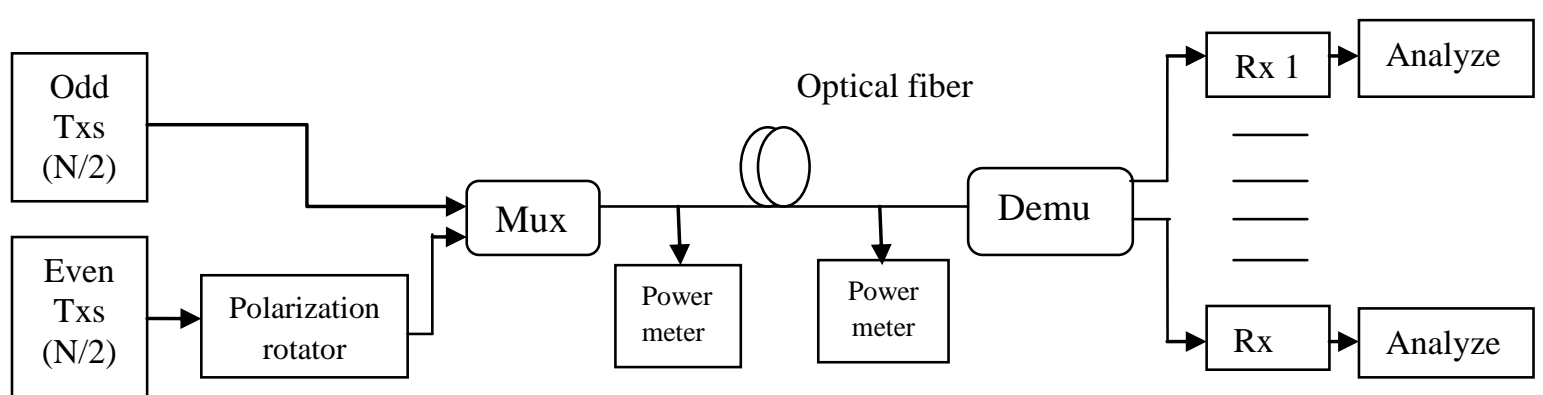

Figure: 1 Block diagram for a polarization interleaved system

\section{Simulation Modelling}

The simulation setup of proposed polarization-based DWDM system modeled using Optisystem version 12 simulation software is shown in the Fig.2. The transmitter section outputs a narrow linewidth optical signal which is modulated with the data source. To drive the simulation, a pseudorandom binary sequence is used. A Continuous Wave (CW) Lorentzian laser which is a simple laser model ,that considers only the phase noise is used for transmission. Among the 8 channels, a polarization rotator is employed at alternate channels. The CW laser operates at a frequency of $193.1 \mathrm{THz}$ with a channel spacing of $0.4 \mathrm{~nm}$. The transmission link includes an $80 \mathrm{~km}$ single mode fiber with dispersion compensation fiber (DCF) and Fiber Bragg Grating (FBG) to compensate for linear effects and an Erbium Doped Fiber Amplifier (EDFA) to overcome attenuation losses.

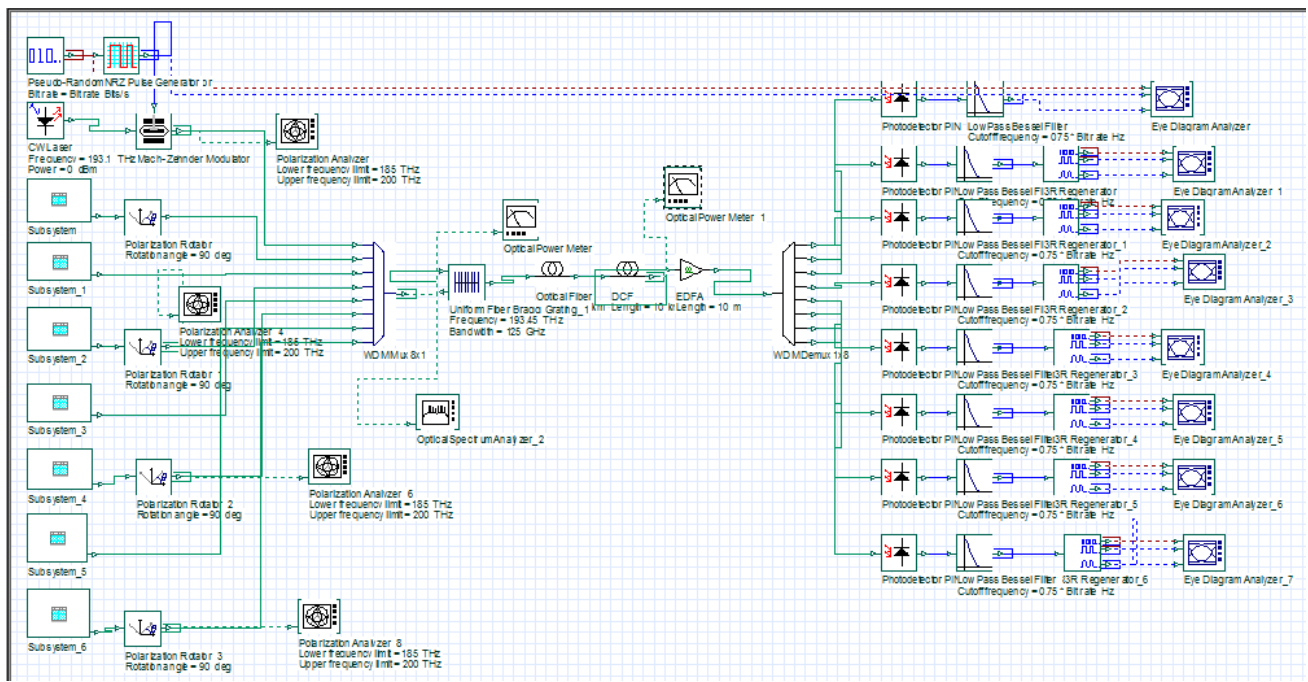

Figure 2. Simulation layout of polarization interleaved system.

The simulation layout for a polarization multiplexed DWDM system is shown in Fig 3. By the concept of polarization multiplexing in WDM two data signals each with $10 \mathrm{~Gb} / \mathrm{s}$, can be transmitted with same wavelength over the fiber simultaneously. In the transmitter side polarization division multiplexing is done using a polarization combiner, and then wavelengths multiplexed using a multiplexer. Similarly, at the receiver side after demultiplexing the wavelengths the signals have to be recovered by polarization demultiplexing using polarization splitter. The signals are detected using a PIN photodiode and electrical filter. The results are estimated using the eye diagrams obtained from the eye diagram analyzers at each of the 8 channels. 


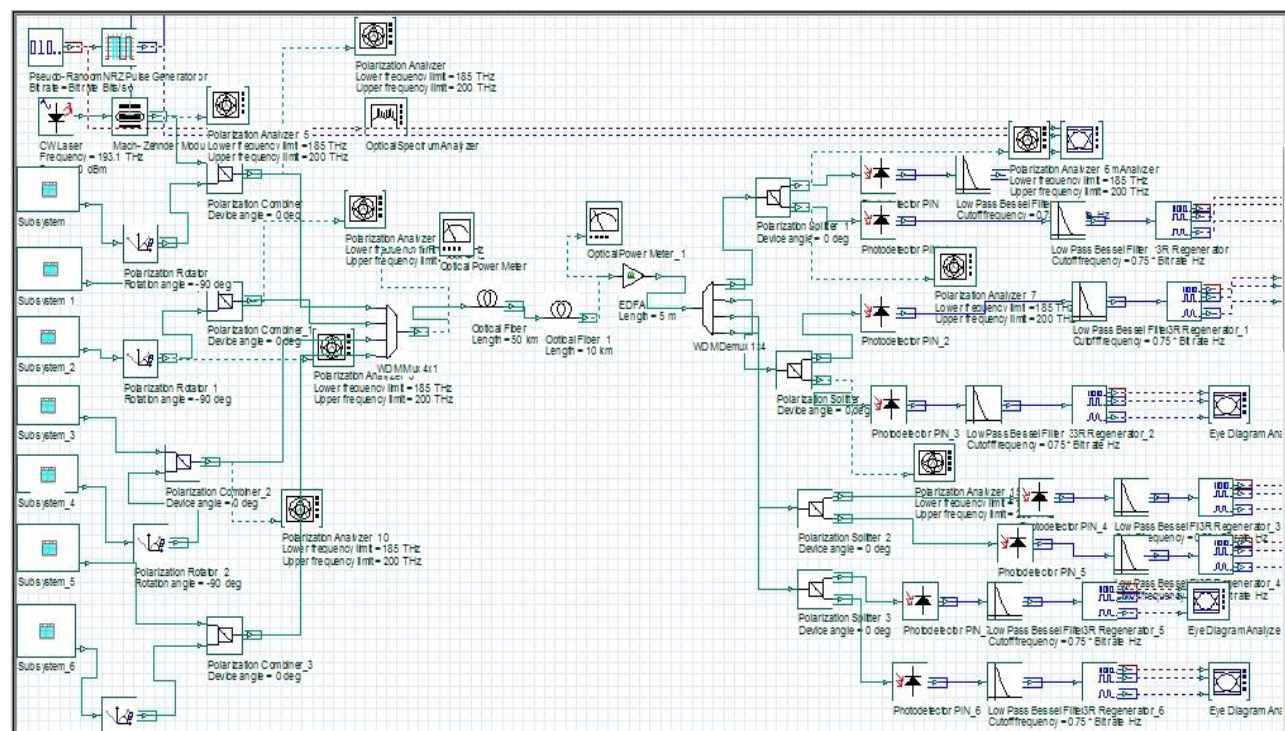

Figure 3. Simulation layout of a polarization multiplexed DWDM system.

\section{Results And Discussions}

The results obtained from eye diagram analyzers of Optisystem version 12 is shown in the Fig 4.For the case of DWDM system with adjacent channel polarization the Quality factor obtained is 5.61526 and for DWDM system with polarization multiplexing the Q factor is 5.35382.Compared to a normal DWDM system with no polarization concept the polarization based system shows an improvement in the performance of the system.

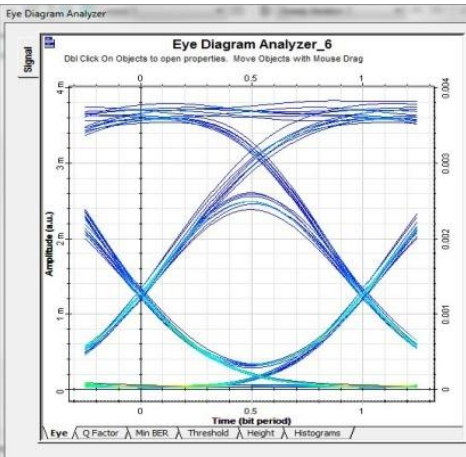

(a)

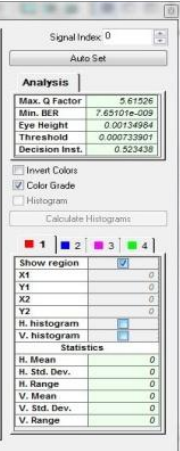

(b)

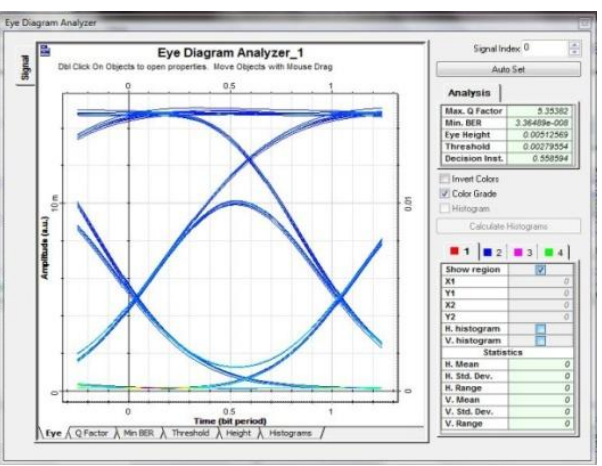

(b)

Figure 4.Eye diagrams obtained for two polarization based systems with a) polarization interleaving b) polarization multiplexing.

\section{Conclusion}

In this paper a DWDM system with polarization based system is analyzed in order to mitigate the nonlinear effects within the fiber channel. The improvement in $\mathrm{Q}$ factor indicates the decrease in optical power loss along the channel since the energy leakage to adjacent channels is minimized. The polarization multiplexed DWDM system serves as a tradeoff as more components have to be used to transmit data simultaneously with different polarization. Hence, the performance can be further improved by using Polarization Multiplexed QPSK modulation in the transmitter section of DWDM.

\section{References}

[1]. Mandeep Kaur, Parminder Singh and Navtej Singh "Analysis of Transmission Formats in Dense Wavelength Division Multiplexing (DWDM) System" IJEIT Vol. 1 No. 1 Oct 2009.

[2]. Sabapathi Tand Nanthini Devi B. S. "Combating SRS and FWM in an Optical Fiber through Unequal Spacing and Dispersion" International Journal of Scientific Engineering and Technology (ISSN : 2277-1581)

[3]. Volume No.2, Issue No.6, pp : 545-549 1 June 2013.

[4]. Alberto Bononi, Armando Vannucci, A. Orlandini, E. Corbel, S. Lanne, and S. Bigo, "Degree of Polarization Degradation Due to Cross-Phase Modulation and Its Impact on Polarization-Mode Dispersion Compensators" Journal of Lightwave Technology, Vol. 21, No. 9, September 20031903

[5]. Peter J. Winzer "Modulation and multiplexing in optical communication systems" IEEE LEOS NEWSLETTER Volume 23,No 1 February 2009

[6]. Jagjit Singh Malhotra , Alok Kumar "Impact of Adjacent Channel Polarization in Long Haul DWDM

[7]. Optical Communication Link" International Journal of Information \& Communication Technology Vol. 1, Issue 1, 2013 